\title{
C-reactive protein velocity discriminates between acute viral and bacterial infections in patients who present with relatively low CRP concentrations
}

\author{
Daniel Bernstein ${ }^{\dagger}$, Dan Coster ${ }^{2 \dagger}$, Shlomo Berliner ${ }^{3}$, Itzhak Shapira ${ }^{3}$, David Zeltser ${ }^{3}$, Ori Rogowski ${ }^{3}$, Amos Adler ${ }^{4}$, \\ Ora Halutz ${ }^{4}$, Tal Levinson ${ }^{3,5^{*}}$ (D), Omri Ritter ${ }^{6}$, Shani Shenhar-Tsarfaty ${ }^{3}$ and Asaf Wasserman ${ }^{3}$
}

\begin{abstract}
Background: To assess the utility of C-reactive protein (CRP) velocity to discriminate between patients with acute viral and bacterial infections who presented with relatively low CRP concentrations and were suspected of having a bacterial infection.

Methods: We analyzed a retrospective cohort of patients with acute infections who presented to the emergency department (ED) with a relatively low first CRP measurement (CRP1) $\leq 31.9 \mathrm{mg} / \mathrm{L}$ and received antibiotics shortly after. We then calculated C-reactive protein velocity (CRPV), milligram per liter per hour, for each patient based on CRP1 and the second CRP value (CRP2) measured within the first $24 \mathrm{~h}$ since admission. Finally, we compared CRPv between patients with bacterial and viral infections.

Results: We have presently analyzed 74 patients with acute bacterial infections and 62 patients with acute viral infections at the mean age of 80 and 66 years respectively, 68 male and 68 female. CRP1 did not differ between both groups of patients (16.2 \pm 8.6 and $14.8 \pm 8.5$ for patients with viral and bacterial infections respectively, $\mathrm{p}$ value = 0.336). However, the CRP2 was significantly different between the groups (30.2 \pm 21.9 and $75.6 \pm 51.3$ for patients with viral and bacterial infections respectively, p-value $<0.001)$ and especially the CRPv was much higher in patients with acute bacterial infections compared to patients with acute viral infections $(0.9 \pm 1.2$ and $4.4 \pm 2.7$ respectively, p-value $<0.001)$.

Conclusion: CRPv and CRP2 are useful biomarkers that can discriminate significantly between patients who present with acute bacterial and viral infections, and relatively low CRP concentration upon admission who were suspected of having a bacterial infection.
\end{abstract}

Keywords: Bacterial, Viral, C-reactive protein, Velocity, Differential diagnosis, Infection

*Correspondence: tallevinson32@yahoo.com

${ }^{\dagger}$ Daniel Bernstein and Dan Coster contributed equally to this work

${ }^{3}$ Departments of Internal Medicine "C", "D" and "E", Tel-Aviv Sourasky Medical Center, and Sackler Faculty of Medicine, Tel-Aviv University, 6 Weizmann Street, 64239 Tel Aviv, Israel

Full list of author information is available at the end of the article

\section{Background}

C-reactive protein (CRP) is an established biomarker for the assessment of inflammation and its severity in patients who present to medical facilities $[1,2]$ and can be used as a reliable, fast and inexpensive indicator of the infection type [3]. regulation or exceeds the permitted use, you will need to obtain permission directly from the copyright holder. To view a copy of this licence, visit http://creativecommons.org/licenses/by/4.0/. The Creative Commons Public Domain Dedication waiver (http://creativeco mmons.org/publicdomain/zero/1.0/) applies to the data made available in this article, unless otherwise stated in a credit line to the data. 
Several studies showed that higher CRP values strengthen the differential diagnosis of acute bacterial infections over acute viral ones. However, the exact cut-off is still disputable and many different CRP values were suggested to indicate a bacterial infection (such as 10, 20, 40, 60 and $100 \mathrm{mg} / \mathrm{L}$ ) [1, 4-7]. This highlights the difficulty for physicians in using CRP for the diagnosis of patients with bacterial infections presenting with relatively low CRP concentrations. Previous studies showed that patients who presented with relatively low CRP values constitute a substantial fraction of the admissions to the emergency department (ED) with acute infectious disease and have a high potential to deteriorate quickly. Wassermann et al. showed that $19.4 \%$ of patients admitted to ED with relatively low CRP levels $(<31.9 \mathrm{mg} / \mathrm{L})$ and their discharge diagnosis was sepsis, died within 1 week of hospitalization [8]. Levinson et al. presented that $6 \%$ of patients diagnosed with gram-negative bacteremia and had an initial low CRP measurement $(<30 \mathrm{mg} / \mathrm{L})$ died within 1 week of hospitalization [9]. Feigin et al. suggested that presentation to the internal medicine department with a very low concentration of CRP $(<0.05)$ does not exclude the existence of significant acute morbidities [10]. These studies emphasize the importance of early diagnosis in acutely ill patients presenting with relatively low CRP values.

Studies have shown the usefulness of sequential measurements of CRP as a tool in the follow-up of different conditions such as community-acquired Pneumonia, ventilator-associated pneumonia, bloodstream infection and sepsis $[9,11]$. The ratio between consecutive CRP measurements was suggested to assess the patient prognosis or the appropriateness of antibiotic therapy. Other studies tried to use the kinetics of CRP for the microbial etiology diagnosis, specifically in the early discrimination between acute viral and bacterial infections. Our own study by Paran et al. presented the possibility to use CRP velocity as a diagnostic tool in patients with bacterial febrile diseases [12]. More recently, Coster et al. presented the usefulness of CRP trend, based on the rate of change between the first and second CRP measurements taken after admission, demonstrating the potential utility of this biomarker for the differentiation of viral versus bacterial infections [13]. These findings strengthen our hypothesis that utilizing CRP kinetics at the first hours upon admission to the medical center can improve the distinction between acute viral and bacterial infections, as the rate of CRP concentration change holds more information regarding the inflammatory process over time than a single CRP measurement. Given good discrimination by CRP kinetics among patients presenting with relatively low CRP values, which are not indicative of infection type, could help in avoiding misuse of antibiotics.

\section{Methods \\ Study design and setting}

We conducted a historical cohort study of patients admitted through the ED in Tel Aviv Sourasky Medical Center (TASMC), Israel, between April 2011 and March 2019. We proposed investigating our assumption by looking at patients with viral and bacterial infections who presented with relatively low first CRP measurement (CRP1) and received antibiotics soon after. This cohort enabled us to evaluate the differentiation ability of CRPv when the CRP1 upon admission was not indicative of infection type, and yet, physicians decided on treatment with antibiotics. This clinical scenario reflects a setting where a differentiation biomarker could be highly significant and prevent inappropriate antibiotic treatment. Hospital records were reviewed manually in order to apply the exclusion criteria.

\section{Patients}

All the patients had a relatively low $\mathrm{CRP} 1 \leq 31.9 \mathrm{mg} / \mathrm{L}$, upon admission and a positive lab test for either a viral or bacterial infection. This relatively low CRP concentration cut-off represents the mean CRP of apparently healthy individuals +3 standard deviation. It was suggested by Wasserman et al., based on a relatively large cohort of individuals who were screened as part of a routine annual check-up [8].

\section{Inclusion criteria}

We included all patients admitted to the general ED in TASMC, Israel, who are adults aged $\geq 18$. We could not include younger patients in this study because they are admitted to a separate pediatric ED in TASMC, and we did not have access with the current Helsinki approval to their medical records. Bacterial infections were identified by a positive blood culture for a single bacteria species that is likely to cause infection and not be a contaminant (defined as blood culture results of Diphtheroids spp, Coagulase-negative Staphylococcus, Streptococcus viridans group and a result of Gram-positive bacilli). Viral infections were identified by either a positive PCR for a virus or an immunoglobulin test indicative of a single viral species.

To link between the CRP measurement and the infectious diagnosis we only included patients whose positive blood culture was taken within 1 day or positive viral test within 5 days of the first CRP measurement. The time difference between CRP1 to the second CRP measurement (CRP2) was less than $24 \mathrm{~h}$. 


\section{Exclusion criteria}

Patients who were not treated with antibiotics between CRP1 and CRP2 or received antibiotics during the $24 \mathrm{~h}$ before admission.

Patients with co-infections, defined as having both a bacterial and viral positive test.

Patients with active malignancy or active inflammatory disease, for example, Systemic lupus erythematosus, Inflammatory bowel disease etc. Patients who were treated with anti-inflammatory medications or immunocompromised patients. Pregnant women and patients with missing medical records were excluded as well (Fig. 1).

\section{Laboratory methods}

Wide range (WR) CRP measurements were done by ADVIA 2400 (Siemens Healthcare Diagnostics Inc., Tarrytown, NY, USA). The ADVIA ${ }^{\circledR}$ Chemistry WR-CRP method measures CRP in the serum and plasma by a latex-enhanced immunoturbidimetric assay.

\section{Computational methods}

CRP velocity (CRPv) was defined as the difference between the second CRP measurement (CRP2) and the first measurement (CRP1), divided by the time difference between the tests ( $\triangle \mathrm{t}$ CRP1 to CRP2, hours).

$$
C R P v=\frac{C R P 2-C R P 1}{\Delta \mathrm{t} C R P 1 \text { to } \mathrm{CRP} 2}
$$

Estimated CRP (eCRP) was defined as expected CRP in apparently healthy individuals based on age and sex. eCRP was calculated based on the data of Tel Aviv Medical Center Inflammation Survey. First, we divided the cohort by sex and then we calculated the mean CRP by age groups of 5 years (starting with individuals 20-25 years old to 75 years). There was a relatively small number of subjects in the male and female group above the age of 75 , hence, we calculated their mean CRP concentration and considered it as the eCRP of all the patients above the age of 75 years. In addition, the healthy cohort did not include subjects younger than 20 years, so we clinically estimated the eCRP value of this age group (Our cohort had only one female patient younger than 20 and her eCRP value was $1.5 \mathrm{mg} / \mathrm{L}$ ). The eCRP values of each age group are reported on Additional file 1: Table S1. The purpose of calculating eCRP is to estimate the CRP level of the patient in the healthy condition in the initiation of his pathological course. Estimated CRP velocity $(\mathrm{eCRPv})$ was defined as the difference between CRP1 and eCRP divided by the time difference between the beginning of symptoms and CRP1 test ( $\Delta \mathrm{t}$ onset of symptoms to CRP1, hours) was defined as eCRPv.

The exact timing from the onset of symptoms was estimated based on the medical record of each patient's admission file.

$$
\mathrm{eCRPv}=\frac{C R P 1-\mathrm{eCRP}}{\Delta \mathrm{t} \text { between CRP1 and symptoms onset }}
$$

The recorded antibiotic time was used to calculate the $\Delta t$ Antibiotic; the time difference between the CRP1 measurement and antibiotic administration.

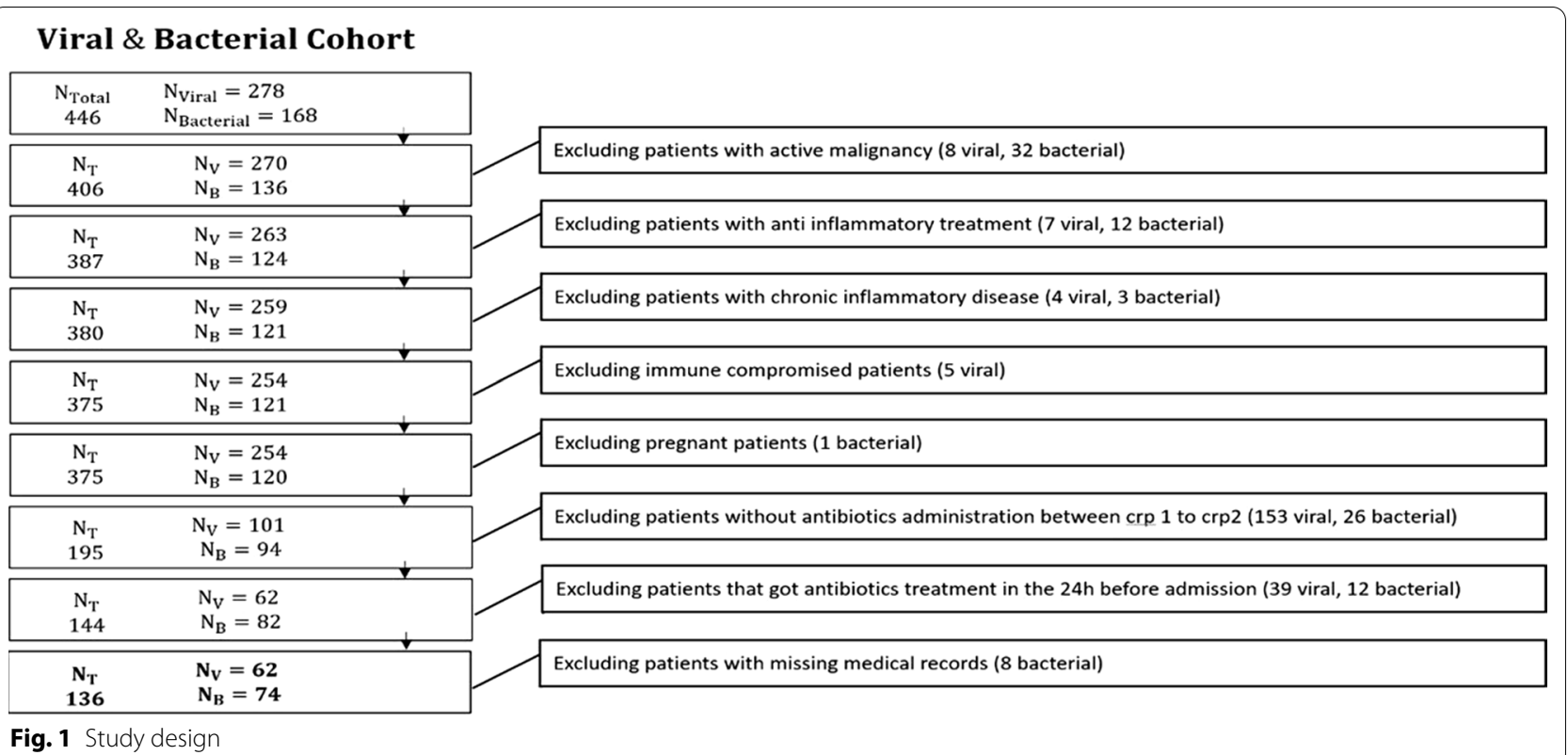




$$
\Delta \text { t Antibiotic }=t_{\text {Antibiotic Administration }}-t_{C R P 1}
$$

For cases in which the administration time of antibiotic in ED was missing, we considered the time of admission to ward to be the antibiotic administration time.

\section{Statistical analysis}

Categorical variables were reported as numbers and percentages. The continuous variables were reported as means with standard deviations. To compare the distributions of different features on the bacterial and the viral groups we used the non-parametric test of Mann-Whitney $(\mathrm{MW})$. In order to compare the sex categorical feature, we used the Pearson's Chi-squared test $\left(\mathrm{Chi}^{2}\right)$. To test the performance of the numerical parameters (e.g., eCRP, CRP1, CRP2, eCRPv, CRPv, etc.) as biomarkers for classification, we used receiver operator characteristic curve (ROC) analysis and calculated the area under the curve (AUC) and for each parameter. Standard nonparametric bootstrapping of 1000 samples was used to generate the $95 \%$ confidence intervals. The statistical analysis was performed using the Python programming language version 3.5.2 and the packages SciPy, NumPy and scikit-learn.

\section{Results}

Applying our inclusion criteria our study covered 446 adult patients, 168 with bacterial infections and 278 with viral infections. Applying our exclusion criteria, the final cohort for analysis covered 136 patients, 74 (54\%) with bacterial infection and $62(46 \%)$ with viral infection. Their characteristics are presented in Table 1. Specific bacterial and viral types are reported in Additional file 1: Tables S2 and S3 respectively.

Patients with bacterial infections were older than patients with viral infections and had similar percent of females. The time difference between CRP1 measurements and antibiotic administration time ( $\Delta \mathrm{t}$ Antibiotic) was similar between patients with viral and bacterial infections. The time differences between the onset of symptoms to the CRP1 measurement ( $\Delta \mathrm{t}$ onset of symptoms to CRP1) was greater in patients with viral infections, compared to patients with bacterial infections $(72.7 \pm 104.0$ and $29.1 \pm 60.8$ respectively, $\mathrm{p}$-value $<0.001$, AUC 0.75, CI 0.67-0.82). The eCRPv was greater in patients with bacterial infections compared to patients with viral infections $(1.2 \pm 1.1$ and $0.8 \pm 1.6$ respectively, p-value $<0.001$, AUC 0.7, CI 0.62-0.77). Patients with bacterial infections had higher CRP2 than patients with viral infections had $(75.6 \pm 51.3$ and $30.2 \pm 21.9$ respectively, p-value $<0.001$, AUC 0.77, CI 0.70-0.84). CRPv was significantly greater in patients with bacterial infections compared to patients with viral infections $(4.4 \pm 2.7$ and $0.9 \pm 1.2$ respectively, p-value $<0.001$, AUC $0.86 \mathrm{CI}$ 0.79-0.91) (Table 1). CRPv had the highest classification quality in comparison to all the others classification parameters.

We evaluated the difference of CRPv levels between two age groups, one group above the age of 79 (median age) and the other below it. CRPv was statistically

Table 1 Demographic characteristics of the bacterial and viral groups

\begin{tabular}{|c|c|c|c|c|c|c|}
\hline \multicolumn{7}{|l|}{ Table of characteristics } \\
\hline Name & $\begin{array}{l}\text { Viral } \\
N=62\end{array}$ & $\begin{array}{l}\text { Bacterial } \\
N=74\end{array}$ & AUC & $\mathrm{Cl}$ & $\begin{array}{l}\text { MW } \\
\text { p value }\end{array}$ & $\begin{array}{l}\text { ChiSquare } \\
\text { p value }\end{array}$ \\
\hline Age (years) & $66.6 \pm 18.4$ & $80.2 \pm 13.7$ & 0.74 & - & $<0.001$ & - \\
\hline Women (\%) & $58.1 \%$ & $43.2 \%$ & 0.57 & - & - & 0.12 \\
\hline$\Delta$ t Antibiotic (h) & $5.4 \pm 5$ & $4.2 \pm 4.2$ & 0.58 & - & 0.097 & - \\
\hline eCRP & $2.9 \pm 0.3$ & $2.8 \pm 0.2$ & 0.58 & - & 0.116 & - \\
\hline CRP1 (mg/L) & $16.2 \pm 8.6$ & $14.8 \pm 8.5$ & 0.55 & $0.46-0.62$ & 0.336 & - \\
\hline $\begin{array}{l}\Delta \text { t onset of symptoms to } \\
\text { CRP1 (h) }\end{array}$ & $72.7 \pm 104$ & $29.1 \pm 60.8$ & 0.75 & $0.67-0.82$ & $<0.001$ & - \\
\hline eCRPv (mg/L/h) & $0.8 \pm 1.6$ & $1.2 \pm 1.1$ & 0.7 & $0.62-0.77$ & 0.001 & - \\
\hline CRP2 (mg/L) & $30.2 \pm 21.9$ & $75.6 \pm 51.3$ & 0.77 & $0.70-0.84$ & $<0.001$ & - \\
\hline$\triangle \mathrm{t}$ CRP1 to CRP2 (h) & $14.9 \pm 5.9$ & $13.1 \pm 6.4$ & 0.58 & - & 0.108 & - \\
\hline CRPv (mg/L/h) & $0.9 \pm 1.2$ & $4.4 \pm 2.7$ & 0.86 & $0.79-0.91$ & $<0.001$ & - \\
\hline
\end{tabular}

Values are mean $\pm S D$, \% for women, MW p-value of the Mann-Whitney test, Chi2p value for the Pearson's Chi-squared test. In the AUC computation, the positive class was bacterial infection for these parameters-eCRPv, CRP2, CRPv and viral infection for these parameters-CRP1, $\Delta t$ onset of symptoms to CRP1, $\triangle t$ CRP1 to CRP2. CRP1 and CRP2: the first and second CRP consecutive measurements after admission. $\triangle \mathrm{t}$ Antibiotic: the time difference between CRP1 and antibiotic administration time. eCRP: estimated CRP is the average CRP concentration in the healthy population compatible by age and sex. $\triangle \mathrm{t}$ Symptoms onset to CRP1: the time difference between onset of symptoms and CRP1. eCRPv: the difference eCRP and CRP1 divided by $\triangle t$ onset of symptoms to CRP1. CRPv: the difference between CRP1 and CRP2 divided by the time difference between the tests

AUC Area under the curve, $C R P C$-reactive protein, $C R P V$ C-reactive protein velocity, eCRPv Estimated C-reactive protein velocity 
significant between the two age groups. After the stratification of the age groups to patients with viral and bacterial infections, the CRPv was not found to be statistically significant (reported in Additional file 1: Table S4). Next, we evaluated the difference of CRPv levels between genders and it was not found to be statistically significant. We also stratified genders to the former age groups (above and below the median age of 79 years) and it was not found to be statistically significant (reported in Additional file 1: Table S5). Lastly, we stratified gender to patients with viral and bacterial infections and it was found to be statistically insignificant (reported in Additional file 1: Table S6).

\section{CRPv performance for different $\Delta t$ values in comparison to CRP1, eCRPv and CRP2}

We evaluated the impact of the time difference between CRP1 and CRP2 on the classification quality in order to search for the parameter with the highest AUC on the earliest time point. We did this by reanalyzing the subgroups of patients with $\triangle$ tCRP1 to CRP2 $\leq X$ hours, for $\mathrm{X}$ values higher than $8 \mathrm{~h}$ (subgroups of intervals shorter than this were too small for analysis). The CRPv remained superior to all the other parameters in any time gap between the CRP1 and CRP2 measurements (Fig. 2).

\section{Discussion}

To the best of our knowledge, this is the first study to show the usefulness of CRPv as a biomarker to distinguish between patients with acute viral and bacterial infections, who presented with relatively low CRP1 and received antibiotic treatment soon after. Our study shows that it was only CRP2, as well as CRPv that could significantly discriminate between both types of infection. Therefore, CRPv could have been used in guiding antibiotic treatment among patients with relatively low CRP1 concentrations, which and are not indicative of infection type, but make up a significant portion of ED admissions for acute infectious diseases [8]. One of strengths in our study is that we included only patients treated with antibiotics, using it as a marker for high clinical suspicion of bacterial infection. The use of antibiotic treatment in patients with proven viral disease shows that the treating physicians got no indication of infection type using the result of the first CRP test. By looking at the second CRP, taken only several hours later, they could have obtained much more information and possibly avoided antibiotic misuse.

In addition, we could show that by calculating the eCRPv upon admission, we could significantly increase the ability to differentiate between viral and bacterial infections already during the presentation to the medical facility. We do believe that this is an additional significant

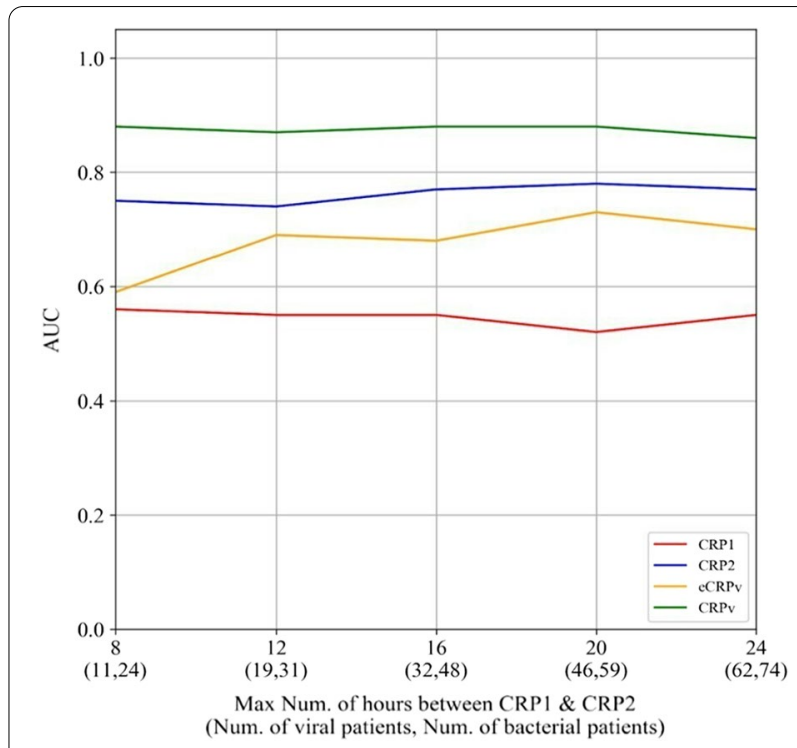

Fig. 2 CRPv performance for different $\Delta t$ values in compare to CRP1, eCRPv and CRP2. AUC Area under the curve, CRP C-reactive protein, $C R P \vee C$-reactive protein velocity, eCRPv Estimated C-reactive protein velocity

finding of the present study as the information of duration of symptoms at the time of CRP1 measurement is readily accessible to the treating physician and can also help in guiding antibiotic treatment.

The CRP2 and especially CRPv further helped to differ between bacterial and viral infections, with a rapid rise in CRP associated with bacterial infections. Our findings demonstrate that for each time point examined, using CRP velocity is more informative than the absolute value of the CRP measurement (e.g., the AUC of eCRPv is higher than the AUC of CRP1, and the AUC of CRPv are higher than the AUC of CRP2).

In the present study, we have calculated only the rise of CRP at the early hours following admission and not the trend as described by Coster et al. [13]. CRP trend uses all the changes in CRP concentration, including CRP decrements, which can also help in the process of differentiation between both types of infection. However, this type of analysis (CRP trend) is less practical for patients who present with relatively low CRP concentrations. It might be more relevant in individuals who present with high CRP concentrations.

Previous studies indicated that CRP levels among healthy individuals are associated with age and gender $[14,15]$ while a recent large study did not support that [16]. Hence, we evaluated their impact on CRPv as potential confounders. We found that $\mathrm{CRPv}$ was higher among adults older than 80 years old however, after stratification to infection type, there was no difference between the 
age groups. It was probably because patients with bacterial infections were older and their CRPv levels were found to be also higher than patients with viral infections (reported in Additional file 1: Table S4). We did not find differences in CRPv among genders, also after stratification to infection type (reported in Additional file 1: Tables S5 and S6 respectively). To the best of our knowledge, we are the first to evaluate the association of CRPv levels with age and gender.

Our study has several limitations, the main one being a retrospective study. In addition, sorting out a certain group of patients that were treated with antibiotics might impose a selection bias. However, this bias is also one of the strengths of our study since it suggests a new paradigm for avoiding antibiotics misuse. Moreover, the fact that all the patients in our cohort were treated with antibiotics could play as a confounding factor. While the patients with bacterial infections are supposed to be affected by the antibiotic treatment, the patients with viral infections are inherently unaffected. Considering Coster et al. results [13], including also patients who were not treated with antibiotics and showed that CRPv could still distinguish viral from bacterial infections, we may assume this confounding factor weakens. Next, we plan to expand our research and evaluate our findings with more diverse groups such as viral who got appropriate treatment and bacterial who did not get appropriate treatment. In addition, some possible confounders of CRP levels were not recorded in our dataset, such as race [15]. In our subsequent studies, we wish to evaluate them prospectively. To the best of our knowledge, the effect of these variables has not been researched in association with CRP kinetics.

Despite the limitations mentioned above, we believe our findings may assist in an actual clinical problem. In our cohort, the admitting physician decided to treat all patients with antibiotics, although $45 \%$ of them were later discovered to have had a viral diagnosis, showing the limits of a single relatively low CRP measurement to reduce antibiotic misuse. With the growing problem of antibiotic resistance [17-19], simple tools such as using the kinetics of CRP as shown in our study should be further explored.

\section{Conclusions}

In patients presenting with relatively low CRP concentrations but high clinical suspicion of bacterial infection, calculating the rate of rise to first CRP and the dynamics to a second CRP measurement helps in differentiating between bacterial and viral etiologies.

\section{Abbreviations}

AUC: Area under the curve; CRP: C-reactive protein; eCRP: Estimated C-reactive protein; CRPv: C-reactive protein velocity; eCRPv: Estimated C-reactive protein velocity; ED: Emergency department; WR-CRP: Wide range C-reactive protein; MW: Mann-Whitney; ROC: Receiver operator characteristic curve.

\section{Supplementary Information}

The online version contains supplementary material available at https://doi. org/10.1186/s12879-021-06878-y.

Additional file 1. Tables S1-S6.

\section{Acknowledgements}

This study was conducted as part of the requirements for graduation from the medical school of the faculty of health sciences, Ben-Gurion University of the Negev, Israel.

Dan Coster was supported, in part, by the Edmond J. Safra Center fellowship for Bioinformatics at Tel Aviv University.

\section{Authors' contributions}

DB, DC: Data collection, data analysis, writing; SB: Study design, writing, review and editing. IS, DZ, OR: Study design, validation. AA: data curation, validation. $\mathrm{OH}, \mathrm{OMR}$ : Data collection, validation. TL: Writing, validation, review and editing. SST: Data analysis, writing. AW: Data analysis, data curation, writing. All authors read and approved the final manuscript.

\section{Funding}

This research did not receive any specific grant from funding agencies in the public, commercial, or not-for-profit sectors.

\section{Availability of data and materials}

The data that support the findings of this study are available from the corresponding author, [T.L.], upon reasonable request.

\section{Declarations}

Ethics approval and consent to participate

The study was approved by the Tel-Aviv Sourasky Helsinki committee and conformed to the principles outlined in the declaration of Helsinki (Numbers 0491-17-TLV). The data that support the findings of this study are available on request from Prof. Shlomo Berliner. The data are not publicly available due to privacy and ethical restrictions. After data retrieval, the analysis was conducted on anonymised data.

\section{Consent for publication}

Not applicable.

\section{Competing interests}

The authors declare that they have no competing interests.

\section{Author details}

'Joyce \& Irving Goldman Medical School, Faculty of Health Sciences, BenGurion University of the Negev, Beersheba, Israel. ${ }^{2}$ Blavatnik School of Computer Science, Tel-Aviv University, Tel-Aviv, Israel. ${ }^{3}$ Departments of Internal Medicine "C", "D" and "E", Tel-Aviv Sourasky Medical Center, and Sackler Faculty of Medicine, Tel-Aviv University, 6 Weizmann Street, 64239 Tel Aviv, Israel. ${ }^{4}$ Clinical Microbiology Laboratory, Tel-Aviv Sourasky Medical Center, Tel-Aviv, Israel. ${ }^{5}$ Infectious Diseases Unit, Tel-Aviv Sourasky Medical Center, Tel-Aviv, Israel. ${ }^{6}$ Department of Emergency Medicine, Tel-Aviv Sourasky Medical Center, Tel-Aviv, Israel

Received: 10 January 2021 Accepted: 15 November 2021

Published online: 04 December 2021

References

1. Gabay C, Kushner I. Acute-phase proteins and other systemic responses to inflammation. N Engl J Med. 1999;340(6):448-54. 
2. Pepys MB, Hirschfield GM. C-reactive protein: a critical update. J Clin Invest. 2003;111(12):1805-12.

3. Lelubre C, Anselin S, Boudjeltia KZ, Biston P, Piagnerelli M. Interpretation of $C$-reactive protein concentrations in critically ill patients. Biomed Res Int. 2013;2013:11

4. Lubell Y, Blacksell SD, Dunachie S, Tanganuchitcharnchai A, Althaus T, Watthanaworawit W, et al. Performance of C-reactive protein and procalcitonin to distinguish viral from bacterial and malarial causes of fever in Southeast Asia. BMC Infect Dis. 2015;15(1):511.

5. Chan Y-L, Liao H-C, Tsay P-K, Chang S-S, Chen J-C, Liaw S-J. C-reactive protein as an indicator of bacterial infection of adult patients in the emergency department. Chang Gung Med J. 2002;25:437-82.

6. Butler CC, Gillespie D, White P, Bates J, Lowe R, Thomas-Jones E, et al. C-Reactive protein testing to guide antibiotic prescribing for COPD exacerbations. N Engl J Med. 2019;381 (2):111-20. https://doi.org/10.1056/ NEJMoa1803185.

7. Woodhead M, Blasi F, Ewig S, Garau J, Huchon G, leven M, et al. Guidelines for the management of adult lower respiratory tract infections-Full version. Clin Microbiol Infect. 2011;17:E1-59.

8. Wasserman A, Karov R, Shenhar-Tsarfaty S, Paran Y, Zeltzer D, Shapira I, et al. Septic patients presenting with apparently normal C-reactive protein: a point of caution for the ER physician. Medicine (Baltimore). 2019;98(2):e13989.

9. Levinson T, Tamir N, Shenhar-Tsarfaty S, Paran Y, Zeltzer D, Shapira I, et al. The potential benefit of a second C-reactive protein measurement in patients with gram-negative bacteraemia presenting to the emergency medicine department. Biomarkers. 2020;25:533-8.

10. Feigin E, Levinson T, Berliner S, Zeltser D, Itzhak S, Shenhar-Tsarfaty S, et al. Patients who are admitted to the Department of Internal Medicine with a very low C-reactive protein concentration. Eur J Inflamm. 2021:19:205873922110473. https://doi.org/10.1177/20587392211047303.

11. Lobo SM. Sequential C-reactive protein measurements in patients with serious infections: does it help? Crit Care. 2012;16(3):130.

12. Paran Y, Yablecovitch D, Choshen G, Zeitlin I, Rogowski O, Ben-Ami R, et al. C-reactive protein velocity to distinguish febrile bacterial infections from non-bacterial febrile illnesses in the emergency department. Crit Care. 2009;13(2):R20.

13. Coster D, Wasserman A, Fisher E, Rogowski O, Zeltser D, Shapira I, et al. Using the kinetics of $\mathrm{C}$-reactive protein response to improve the differential diagnosis between acute bacterial and viral infections. Infection. 2019;48(2):241-8.

14. Tang Y, Liang P, Chen J, Fu S, Liu B, Feng M, et al. The baseline levels and risk factors for high-sensitive $\mathrm{C}$-reactive protein in Chinese healthy population. Immunity Ageing. 2018;15:21.

15. Khera A, McGuire DK, Murphy SA, Stanek HG, Das SR, Vongpatanasin W, et al. Race and gender differences in C-reactive protein levels. J Am Coll Cardiol. 2005;46(3):464-9.

16. Cohen NM, Schwartzman O, Jaschek R, Lifshitz A, Hoichman M, Balicer $R$, et al. Personalized lab test models to quantify disease potentials in healthy individuals. Nat Med. 2021;27(9):1582-91.

17. Nathan C, Cars O. Antibiotic resistance-problems, progress, and prospects. N Engl J Med. 2014;371(19):1761-3.

18. Rossolini GM, Arena F, Pecile P, Pollini S. Update on the antibiotic resistance crisis. Curr Opin Pharmacol. 2014;18:56-60.

19. Hecker MT, Aron DC, Patel NP, Lehmann MK, Donskey CJ. Unnecessary use of antimicrobials in hospitalized patients: current patterns of misuse with an emphasis on the antianaerobic spectrum of activity. Arch Intern Med. 2003;163(8):972-8.

\section{Publisher's Note}

Springer Nature remains neutral with regard to jurisdictional claims in published maps and institutional affiliations.

Ready to submit your research? Choose BMC and benefit from:

- fast, convenient online submission

- thorough peer review by experienced researchers in your field

- rapid publication on acceptance

- support for research data, including large and complex data types

- gold Open Access which fosters wider collaboration and increased citations

- maximum visibility for your research: over 100M website views per year

At BMC, research is always in progress.

Learn more biomedcentral.com/submissions 